\title{
Targetability of hyaluronic acid nanogel to cancer cells: In vitro and in vivo studies
}

\author{
S.S. Pedrosa ${ }^{\mathrm{a}}$, P. Pereira ${ }^{\mathrm{a}}$, A. Correia ${ }^{\mathrm{b}}$, F.M. Gama ${ }^{\mathrm{a}, *}$ \\ a Institute for Biotechnology and Bioengineering, Centre of Biological Engineering, Campus de Gualtar, University of Minho, Braga 4710-05, Portugal \\ b Institute of Molecular and Cell Biology, Rua Campo Alegre, Porto 4099-003, Portugal
}

\section{A R T I C L E I N F O}

\section{Keywords:}

Hyaluronic acid

Cancer nanotechnology

Non-invasive imaging

Non-small cancer lung cells

\begin{abstract}
A B S T R A C T
We have, in previous work developed, characterized and evaluated the biocompatibility of an engineered hyaluronic acid nanogel. Here we assess the targetability of a hyaluronic acid nanogel towards CD44 overexpressing cells, in vitro and in vivo. Results obtained by flow cytometry and confocal fluorescence microscopy shows that nanogel is greatly internalized by non-small cancer lung cells (A549 cells), that overexpress CD44 receptors. The biodistribution and tumor targetability of the nanogel labelled with a nearinfrared (NIR) probe were performed, in mice, through a non-invasive imaging system. Results revealed nanogel high targetability towards an induced subcutaneous A549 tumor. Nanogels pharmacokinetics was evaluated also in healthy animals, and Alexa Fluor 680 labelled nanogel exhibited higher accumulation in liver, kidneys and skin. Also, a comparative biodistribution study was performed, using two NIR imaging probes, Cy5.5 and Alexa Fluor 680.
\end{abstract}

\section{Introduction}

Hyaluronic acid is a naturally occurring polysaccharide, ubiquitous in the human body, widely used in biomedical applications as a conjugate, hydrogel, nanogel and many other applications. Hyaluronic acid demonstrates appealing biological properties such as, biocompatibility, biodegradability and non-antigenic (Arpicco et al., 2014). Among the most attractive features of hyaluronic acid applied to nanomaterials is its potential for active targeting (Goodarzi et al., 2013). The strong affinity of hyaluronic acid for cell surface receptors, namely CD44 and Receptor for Hyaluronan Mediated Motility (RHAMM), has been demonstrated (Goodarzi et al., 2013; Platt and Szoka, 2008). The CD44 receptor is a glycoprotein overexpressed in the cell membrane of numerous cancer cells and important in the metastization process (Arpicco et al., 2014; Goodarzi et al., 2013). We have developed a hyaluronic acid nanogel by grafting a thiolated hydrophobic chain in the polysaccharide backbone. The amphiphilic conjugate self assembles in aqueous environment onto nanostructures. The nanogel has already proven to be crosslinkable by disulfide bond and its physicochemical characterization has already been addressed (Pedrosa et al., 2014). Also, in vitro and in vivo biocompatibility of the engineered nanogel has also been demonstrated (in press). In this work, we intend to assess the nanogel targetability mediated by hyaluronic acid interface towards CD44 overexpressing cancer cells. Non-small cancer lung cells - A549 cell line - express high levels of CD44 receptors (Arpicco et al., 2014; Ganesh et al., 2013) and therefore, were used as a target for nanogel uptake studies. The in vitro cellular uptake of nanogel by A549 cells was assessed by flow cytometry analysis and confocal fluorescence microscopy. Also, we investigated the nanogel targetability in tumor bearing mice - induced with subcutaneous A549 cells tumor. The biodistribution was analysed in a non-invasive real time NIR imaging system, after intravenous administration of probe labelled samples. NIR probes have the advantage of high depth penetration, high fluorescence intensity and amenable self-quenching (Ogawa et al., 2009). Studies have shown that changes in particle size, surface charge, texture and heterogeneity in nanoparticles formulation influence its biodistribution (Adjei et al., 2014). Therefore, we also performed a comparative study of in vivo pharmacokinetics of the HyA nanogel, native HyA and free probe in healthy animals, using two different NIR probes, namely Cy5.5 and Alexa Fluor 680.

\section{Materials}

Sodium hyaluronate (MW.7.46 kDa) was purchased from Lifecore Biomedical (USA). 1-ethyl-3-[3 dimethylaminopropyl]carbodiimide hydrochloride (EDC), cysteamine hydrochloride, trypan blue, 3-(4,5dimethylthiazol-2-yl)-2,5-diphenyl tetrazolium bromide (MTT), hydrogen nicotinamide adenine dinucleotide (NADH) and pyruvate were

\footnotetext{
* Corresponding author.

E-mail address: fmgama@deb.uminho.pt (F.M. Gama).
} 
purchased from Sigma-Aldrich (Italy).

Cy 5.5 hydrazide was purchased from GE Healthcare (UK). Fluorescein-5-thiosemicarbazide and Alexa Fluor $680 \mathrm{C}_{2}$ maleimide acquired from Thermo Fisher Scientific/Life Technologies (USA).

Cell Culture materials were purchased from Biochrom (Germany).

Animals were acquired from Charles River (Germany) and maintained in Molecular Medicine Institute (Lisbon) Rodents Facility.

\section{Methods}

\subsection{Cell lines and cell culture}

Human alveolar adenocarcinoma cells (A549) were maintained in Dulbecco's Modified Eagle's Media (DMEM) supplemented with $10 \%$ ( $v$ / v) of heat-inactivated foetal bovine serum (FBS), $100 \mathrm{IU} / \mathrm{ml}$ penicillin and $0.1 \mathrm{mg} / \mathrm{ml}$ streptomycin. Cystic fibrosis bronchial epithelial cells (CFBE) were cultured in Roswell Park Memorial Institute (RPMI) 1640 Medium supplemented with $10 \%(v / v)$ of heat-inactivated FBS, penicillin and streptomycin. The cells were maintained as a subconfluent monolayer in a humidified atmosphere containing $5 \% \mathrm{CO}_{2}$ at $37^{\circ} \mathrm{C}$.

\subsection{In vitro cell toxicity}

Nanogel toxicity in A549 cell line was assessed through 3-[4,5dimethylthiazol-2-yl]-2,5-diphenyl tetrazolium bromide (MTT) reduction assay (Mosmann, 1983) and lactate dehydrogenase (LDH) release assay (Fotakis and Timbrell, 2006).

\subsubsection{Cell proliferation assay}

A549 cells were seeded in 24-well cell culture plates at a density of $1 \times 10^{4}$ cells per well and left adhering in $0.5 \mathrm{ml}$ of adequate culture medium overnight. HyA-AT nanogel $0.1 \mathrm{mg} / \mathrm{ml}, 0.5 \mathrm{mg} / \mathrm{ml}$ and $1 \mathrm{mg}$ / $\mathrm{ml}$ dispersions were suspended in adequate culture medium, resulting in a 1:5 fresh medium dilution. Untreated cells were used as control of $100 \%$ cell viability and $20 \%$ distilled water control was used to access the effect of water dilution of samples containing the nanogel. A positive control with $20 \%$ of DMSO was used in every analysis. The samples were incubated for 24,48 and $72 \mathrm{~h}$ and the cells metabolic activity is calculated due to the reduction of tetrazolium salt of MTT by mitochondrial succinate dehydrogenase enzymes of metabolically active cells. To which well, $10 \%(v / v)$ of a MTT solution $(5 \mathrm{mg} / \mathrm{ml}$ in PBS) was added and it was incubated at $37^{\circ} \mathrm{C}$ and $5 \% \mathrm{CO}_{2}$ for a period of $4 \mathrm{~h}$. In this period of time, the tetrazolium salt is bioreduced to a formazan product that consists in is dark blue crystals that are insoluble in the culture medium. The supernatant was discarded slowly and the crystals were solubilized in dimethyl sulfoxide and quantified spectrophotometrically at $570 \mathrm{~nm}$. The experiments were performed in triplicates as the results are presented as percentage in which $100 \%$ viability corresponds to the non-treated cells.

\subsection{2. $L D H$ release assay}

Cells were seeded in 12-well plate at a density of the $2 \times 10^{5}$ cells per well and allowed to settle overnight in $0.5 \mathrm{ml}$ of adequate culture medium. Then, cells were treated with nanogel dispersions with a concentration of 0.1 and $1.0 \mathrm{mg} / \mathrm{ml}$ in suitable culture medium and supplementation. Untreated cells were used as control of $100 \%$ cell viability. Another control with $20 \%$ distilled water was used to access the effect of the water dilution of the samples containing the nanogel. A control with $20 \%$ of DMSO was used in every analysis as a positive control. The samples where incubated for $24 \mathrm{~h}$ and after that period each culture medium from every well was collected and centrifuged at $13000 \mathrm{rpm}$ for $1 \mathrm{~min}$ and the cell free supernatant was collected and stored on ice for further analysis - extracellular LDH. Cells were scraped with a Tris solution $(15 \mathrm{mM})$ extracellular and further lysed by sonication. The resulting supernatants were used to quantify the LDH present - Intracellular LDH. An aliquot of extracellular (40 $\mu$ l) or intracellular $(10 \mu \mathrm{l}) \mathrm{LDH}$ were assigned into a microplate and $250 \mu \mathrm{l}$ of the NADH solution $0.31 \mathrm{mM}$ in phosphate buffer $0.05 \mathrm{M}$, pH 7.4 added to each well. Lastly, $10 \mu \mathrm{l}$ of an $8.96 \mathrm{mM}$ piruvate solution in phosphate buffer (substrate solution) was added and immediately afterwards the variation of the absorbance at $340 \mathrm{~nm}$ was read in a microplate spectrophotometer system, as to determine the rate of NADH consumption (slope of the line). LDH leakage was expressed as the ratio between extracellular and total $\mathrm{LDH}$, corresponding the inverse value to the cell membrane integrity. Each experiment was performed in triplicate.

\subsection{In vitro cellular uptake}

The cellular uptake was evaluated by flow cytometry (FCM) analysis, confocal fluorescence microscopy and trypan blue exclusion assays.

\subsubsection{Synthesis of fluorescein labelled nanogel}

Hyaluronic acid nanogel (HyA-AT) synthesis was described elsewhere (Pedrosa et al., 2014). HyA-AT nanogel was conjugated with fluorescein-5-thiosemicarbazide for in vitro cellular studies. Thiosemicarbazide group of the probe reacted with carboxylic group of hyaluronic acid nanogel in presence of EDC as a coupling agent. The theoretical labelling degree, defined by the ratio between probe and the nanogel, was 0.25 . The lack of unconjugated fluorescein was confirmed by ultrafiltration through a $2 \mathrm{kDa}$ MW cut-off membrane. In filtrate and concentrate, probe emission was measured spectrophotometrically and the absence of fluorescence signal was achieved in the last filtrate (Hermanson, 2008; Tanzer et al., 1966).

\subsubsection{Flow cytometry analysis}

Quantitative cellular uptake of nanogel in CD44 expressing (A549) and non-expressing cells (CFBE) was conducted in a Coulter Epics XL Flow Cytometer (Beckman Coulter Inc., Miami, FL, USA). Cells were seeded in 24-well plates at $1.0 \times 10^{5}$ density and incubated overnight to allow attachment. Culture medium was removed and A549 cells were incubated with culture medium containing $0.2 \mathrm{mg} / \mathrm{ml}$ and $0.5 \mathrm{mg} / \mathrm{ml}$ of HyA-AT nanogel, for designated time intervals. Then, CFBE cells were processed similarly using a concentration of $0.5 \mathrm{mg} / \mathrm{ml}$ of nanogel. After 0 and $30 \mathrm{~min}, 1,2,3,5,7$ and $24 \mathrm{~h}$, culture medium was removed and cells were rinsed with PBS. Cells were then harvested by trypsinization, collected with FBS supplemented culture medium and centrifuged at $300 \mathrm{~g}$ for $10 \mathrm{~min}$. Supernatant was rejected and cells were washed with PBS twice and finally resuspended in flow cytometry staining buffer prior to analysis. Cells were gated based on size $v s$ granularity (Forward Scattered/Side Scattered, FSC/SSC) dot plots. The fluorescence due to nanogel internalization was evaluated in FL1 (green channel), where FL1 positive cells had internalized/adhered nanogel. Cell count was set to minimum 20,000 events. Results are expressed as mean fluorescence intensity and the frequencies (or percentage) of cells in gates of interest $(n=5)$.

3.3.2.1. Trypan blue exclusion assay. Trypan blue (TB) is a cell impermeable dye. The exclusion assay is based on the capability of TB to quench green fluorescence (FITC/fluorescein) signal at the cell surface (Vranic et al., 2013; Avelar-Freitas et al., 2014; Keogh et al., 2011). Therefore, TB assay allows us to distinguish between internalized and cell adhering nanogel (Vranic et al., 2013; Gratton et al., 2008). Samples, after being analysed were further incubated with $0.1 \%$ trypan blue for $1 \mathrm{~min}$. Cellular debris and aggregates were excluded by FSC/SSC gating and results were analysed in FL1/FL3 (green/red channels). Results are expressed as mean fluorescence intensity of the FL1 signal $(n=5)$.

\subsubsection{Confocal fluorescence microscopy}

Cellular uptake of HyA-AT nanogel labelled with fluorescein was 
evaluated in CD44 overexpressing cells - A549 cells (McDonald et al., 2008; Roudi et al., 2014; Zhang et al., 2010) and other lung cell line that does not overexpress CD44 receptors - CFBE (Reeves et al., 2011; Reeves et al., 2010; Vandivier et al., 2009).

Cells were seeded on coverslip discs in 24 well plates at $5 \times 10^{5}$ cells/well density. HyA-At-fluorescein $(0.2 \mathrm{mg} / \mathrm{ml}$ concentration) was incubated with cells for $7 \mathrm{~min}$ adequate culture medium and atmosphere conditions. To further assess if the nanogel uptake was mediated by hyaluronic acid receptors, we performed a competitive study with free hyaluronic acid, in A549 cells. So, we pre-incubated A549 cells with $10 \mathrm{mg} / \mathrm{ml}$ free HyA dispersed in culture medium, for $1 \mathrm{~h}$ prior to nanogel addition. The culture medium was removed and replaced with $0.2 \mathrm{mg} / \mathrm{ml}$ of nanogel, and incubated for $7 \mathrm{~h}$. Then, medium was removed and cells were rinsed twice with PBS and fixed with a $2 \%$ para-formaldehyde solution (in PBS). After $20 \mathrm{~min}$, cells were again washed twice with cold PBS. Cell nucleus were stained with 4',6diamidino-2-phenylindole (DAPI, $120 \mathrm{ng} / \mathrm{ml}$ ) for $3 \mathrm{~min}$ at room temperature. Cell seeded coverslips were observed in a confocal laser scanning microscope Leica SP2 AOBS SE (Leica Mycrosystems, Germany).(Avelar-Freitas et al., 2014; Gratton et al., 2008).

\subsection{In vivo spectral imaging studies}

\subsubsection{Animals and treatments}

All animal experiments were performed in compliance with the Portuguese General alimentary and Veterinarian Board (authorization number 006315/27/03/2014, from DGAV-Portugal) and animals were kept and used strictly in accordance with National rules and the European Communities Council Directive (86/609/EEC), for the care and handling of laboratory animals. Balb/cByJ 6-week old male mice and CD1 nude crl: CD1nude 6-week old mice were acquired from Charles River (Germany) and maintained in Molecular Medicine Institute (Lisbon) Rodents Facility. Animals were divided into groups $(n=5)$, for different time point analysis and samples administration. The following samples were tested: nanogels HyA-AT-Cy5.5 and HyAAT-Alexa680; native HyA-Cy5.5 and HyA-Alexa680; free Cy 5.5 and Alexa680. The labelled nanogels (HyA-AT-Cy5.5 and HyA-ATAlexa680) were dispersed in saline solution and filtered through cellulose acetate syringe filter (pore size $0.22 \mu \mathrm{m}$ ) in aseptic conditions and its size distribution analysed by DLS. All the samples were administered intravenously through the tail vein at $5 \mathrm{mg} / \mathrm{kg}$ body weight, as described by several authors (Choi et al., 2010; Choi et al., 2011; Yoon et al., 2013). Samples labelling intensity was compared by $\mathrm{UV} / \mathrm{Vis}$ spectroscopy at probes maximum excitation wavelength to compare their intensity.

\subsubsection{Synthesis of Alexa Fluor labelled hyaluronic acid and hyaluronic acid nanogel}

Alexa Fluor $680 \mathrm{C}_{2}$ maleimide is a thiol reactive conjugate used for NIR probing. Maleimide undergoes an alkylation reaction with sulfhydryl groups to form a thioether bond (Nobs et al., 2003). HyA-AT nanogel is a thiolated hyaluronic acid conjugate and therefore, its thiol group reacts directly with maleimide moiety of the probe. Native hyaluronic acid had to be modified to become reactive towards the maleimide reactive probe. Therefore, similarly to the nanogel synthesis (Pedrosa et al., 2014) cysteamine hydrochloride was grafted by amide bond formation to the carboxylic acid residue of native hyaluronic acid. In brief, HyA-TBA salt was dissolved in DMSO and reacted with cysteamine hydrochloride (2:1) in presence of EDC and NHS (equimolar amounts). The solution was dialysed against a $\mathrm{NaCl}$ solution and then $\mathrm{dH}_{2} \mathrm{O}$ and lyophilized. Resulting thiolated native HyA was conjugated with the probe by the same chemistry.

Hence, $10 \mathrm{mg}$ of nanogel and native HyA reacted with $0.4 \mathrm{mg}(2 \%$ theoretical labelling) of Alexa Fuor $680 \mathrm{C}_{2}$ maleimide in $0.1 \mathrm{M}$ PBS solution ( $\mathrm{pH}$ 7.4). Reaction was allowed to occur overnight at room temperature and protected from light. Afterwards, solutions were dialysed through a $2 \mathrm{kDa}$ cutoff membrane to remove the unreacted probe. Confirmation was obtained by ultrafiltration and UV/VIS analysis of the solutions collected from the upper and lower compartments. Samples were analysed spectrophotometrically at $679 \mathrm{~nm}$. (Gruber et al., 2000) (Hermanson, 2008; Bae et al., 2009; Bae et al., 2008).

3.4.3. Synthesis of cyanine (Cy 5.5) labelled hyaluronic acid and hyaluronic acid nanogel

HyA nanogel and native HyA were labelled with Cy 5.5 - hydrazide. Hydrazide reactive moiety was conjugated with carboxylic groups of HyA-AT nanogel (Hermanson, 2008; Ahn et al., 1987; Na et al., 2007; Luo and Prestwich, 1999) in presence of EDC, as coupling agent. The molar ratio of Cy 5.5 - hydrazide to free carboxylic groups of HyA-AT nanogel and native HyA was 0.25 . EDC was also added at an equimolar ratio to the free carboxylic acid groups of HyA-AT nanogel. The reaction was allowed to occur overnight at room temperature, in the dark. The reaction mixture was thoroughly dialysed (MW cut-off $2000 \mathrm{Da}$ ) against distilled water to remove non-desired reaction products. Also, the absence of unconjugated dye was confirmed by ultrafiltration through a 2 kDa MW cut-off membrane. Samples labelling was assessed spectrophotometrically at $649 \mathrm{~nm}$. Samples labelling was assessed spectrophotometrically at $649 \mathrm{~nm}$. (Ogawa et al., 2009; Berlier et al., 2003) (Ilgin et al., 2010; Kadi et al., 2009).

\subsubsection{Comparison of pharmacokinetics of Cy5.5 and Alexa Fluor 680}

To evaluate the possible differences in biodistribution profile of nanogel when labelled with chemically different NIRF probes, two probes were analysed - Cy 5.5 and Alexa680. Therefore, HyA-AT nanogel and native HyA were labelled with Cy5.5 hydrazide or Alexa680. Free probe was also analysed to infer its in vivo biodistribution profile. Samples were administrated to BALB/c mice at $5 \mathrm{mg} / \mathrm{kg}$ BW through the tail vein. At established intervals (20 min, 2, 7, 24 and $48 \mathrm{~h}$ ) mice were anesthetised with ketamine/medetomidine and whole body NIR fluorescence images were acquired. At the final time point ( $48 \mathrm{~h}$ after sample administration) animals were sacrificed and major organs - liver, lung, spleen, kidney, skin, muscle, heart and brain -, and also blood samples were collected for analysis. NIRF images of dissected organs and blood were obtained and fluorescence intensity quantified by ROI measurement. In vivo real time NIR fluorescence images of the animas were acquired in a Xenogen's IVIS ${ }^{\circledast}$ Lumina Series and Living Image ${ }^{\circledR}$ Software. Imaging was obtained under the following settings: excitation passband of 615-665 nm and emission passband of 695-770 wavelength; exposure time was set to $1 \mathrm{~s}$, pixel binning medium and lens aperture (f/stop) 16.

All values are presented as average fluorescence intensity $\left(\mathrm{p} / \mathrm{s} / \mathrm{cm}^{2} /\right.$ sr) \pm SD for $n=5$ animals.

\subsubsection{Study of in vivo nanogel pharmacokinetics in healthy animals}

We proposed to assess HyA-AT nanogel biodistribution profile in comparison to native HyA after intravenous administration, using free NIR fluorescent probe as control. BALB/c mice were injected in the tail vein with Alexa680 labelled HyA-AT nanogel (HyA-AT-Alexa680) and native HyA (HyA-Alexa680) at $5 \mathrm{mg} / \mathrm{kg}$ BW. At established time points $-5 \mathrm{~min}, 1,8,24$ and $48 \mathrm{~h}$ - animals $(n=5)$ were anesthetised to remain immobile with ketamine $75 \mathrm{mg} / \mathrm{kg}$ BW and medetomidine $1 \mathrm{mg} / \mathrm{kg}$ BW intraperitoneally. In vivo real time NIR fluorescence images of the animas were acquired in a Xenogen's IVIS ${ }^{\circledR}$ Lumina Series and Living Image ${ }^{\circledast}$ Software. After, whole blood was harvested by cardiac puncture and ex vivo organ collection was performed. Major organs - liver, lung, spleen, kidney, skin, muscle, heart and brain - were collected and further analysed. NIRF images of dissected organs and blood were obtained, and fluorescence intensity quantified by ROI measurement. All values are presented as average fluorescence intensity $\left(\mathrm{p} / \mathrm{s} / \mathrm{cm}^{2} / \mathrm{sr}\right) \pm \mathrm{SD}$ for $n=5$ animals. 


\subsubsection{Study of nanogel targetability in tumor xenograft animals}

In vivo tumor targetability of HyA-AT nanogel was assessed in CD1 nude mice with A549 subcutaneous induced tumor. With that intent, HyA-AT nanogel and also native HyA, as a control, were labelled with Alexa Fluor 680. The animal model chosen was Crl: CD1-Foxn1nu mice, due to the lack of thymus once we intended to induce a subcutaneous xenograft tumor. Approximately $5 \times 10^{6}$ A549 cells were suspended in $100 \mu \mathrm{l}$ of saline physiological and Matrigel (BD Biosciences, CA, USA) and were subcutaneously injected into dorsa right side of mice (Ganesh et al., 2013; Choi et al., 2010; Choi et al., 2011; Abbad et al., 2015). Tumor mass was monitored periodically with a caliper and was calculated as $\mathrm{V}=1 / 2$ (length $\times$ width $^{2}$ ). Animals were used in experiment when tumors size reached approximately $100 \mathrm{~mm}^{3}$. As in previous section, HyA-AT-Alexa680 and HyA-Alexa680 labelled materials were administered and at the following time points animals were analysed: $5 \mathrm{~min}, 1,8,24$ and $48 \mathrm{~h}(n=5)$. Prior to image acquisition animals were anesthetised with ketamine/medetomidine combination. After the whole body in vivo image acquisition, whole blood was harvested by cardiac puncture and ex vivo organ collection was performed. Major organs - liver, lung, spleen, kidney, skin, muscle, heart and brain -, also the tumor mass were collected and further analysed. NIRF images of dissected organs, blood and tumor were obtained and fluorescence intensity quantified by ROI measurement. All values are presented as average fluorescence intensity $\left(\mathrm{p} / \mathrm{s} / \mathrm{cm}^{2} / \mathrm{sr}\right) \pm$ SD for $n=5$ animals.

\subsubsection{Ex vivo tissue distribution}

Soon after NIR fluorescence images of mice whole body were obtained, animal's whole blood was collected through cardiac puncture. After, animals were euthanized with a lethal dose of anaesthesia and major organs were collected - liver, lung, spleen, kidney, skin, muscle, heart and brain - and tumor (in tumor targetability assay). Herein, NIR fluorescence images of dissected organs and blood were obtained in the same IVIS ${ }^{\circledR}$ Lumina equipment and fluorescence intensity quantified by ROI measurement. Similar parameters were applied in this analysis as the described in prior section. All values are presented as average fluorescence intensity $\left(\mathrm{p} / \mathrm{s} / \mathrm{cm}^{2} / \mathrm{sr}\right) \pm \mathrm{SD}$ for $n=5$ animals.

\subsection{Statistical analysis}

The results were expressed as mean \pm SD of 3 independent experiments $(n=5)$. Statistical analysis was performed with $t$-test or two-way ANOVA followed by Tukey's comparison test using using GraphPad Prism version 6.00 for Mac OS X, GraphPad Software, La Jolla, California, USA. Significance of the results is indicated according to $P$ values with one, two, three or four of the used symbols (*, \# or + ) corresponding to $P=0.01$ to $0.05 ; P=0.001$ to $0.01 ; P=0.0001$ to 0.001 and $P<0.0001$, respectively).

\section{Results and discussion}

\subsection{In vitro cell toxicity}

Cell proliferation assay using MTT is a colorimetric, easy, fast and safe assay that measures the mitochondrial metabolic activity of viable cells. In Fig. 1a, we observed that the control with $20 \%$ water dilution presented a slightly lower cell growth or activity may be due to the dilution of nutrients of the culture medium. This effect was most noticed in the longer incubation time $(72 \mathrm{~h})$.

Cells metabolic activity was not overall affected by the nanogel presence in comparison to the water diluted control. However, at highest incubation time $(72 \mathrm{~h})$ and nanogel concentration $(1 \mathrm{mg} / \mathrm{ml})$ a significative difference was observed (Fig. 1a). A549 cell line is a human pulmonary adenocarcinoma cell line that overexpresses CD44 receptors - a hyaluronic acid receptor. Therefore, it may be speculated that these receptors mediate a significant nanogel uptake. However, we must mention that the highest concentration tested $(1 \mathrm{mg} / \mathrm{ml})$ is very a
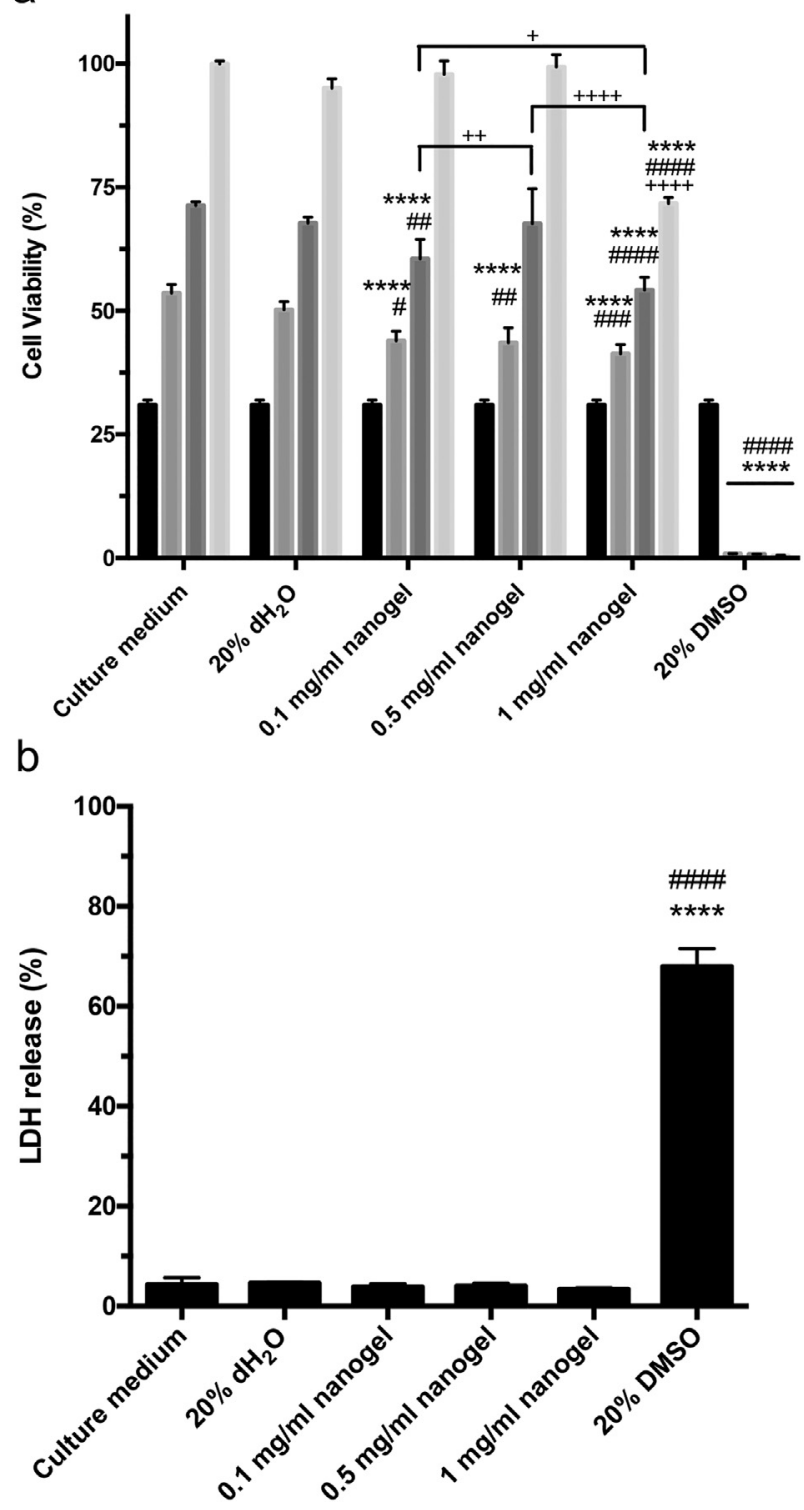

Fig. 1. Nanogel cytotoxic effect on A549 cells was determined by MTT and LDH release assays. a) Cell viability was evaluated by MTT assay at 24,48 and $72 \mathrm{~h}$ and; b) cell membrane integrity by LDH release assay, at $24 \mathrm{~h}$ incubation time.

high, with the intention of really testing the limits of toxicity.

The evaluation of cell membrane integrity was performed by LDH release assay. As shown in Fig. 1b, membrane integrity was preserved at all nanogel concentrations.

Cells were exposure to HyA-AT nanogel at 0.1 to $1 \mathrm{mg} / \mathrm{ml}$ concentration. Non-treated cells referred to as culture medium are considered $100 \%$ cell viability at $72 \mathrm{~h}$. Statistical analysis was performed using a two-way ANOVA and a Tukey's comparison test. Differences between samples and culture medium at any given time point are represented by (*); whereas differences between samples and $20 \% \mathrm{dH}_{2} \mathrm{O}$ dilution control at any given time point are represented by (\#); differences between nanogel concentration are represented by $(+)$.

\subsection{In vitro cellular uptake}

We aim to demonstrate HyA nanogel's ability to actively target cell receptors such as CD44 and RHAMM, overexpressed in numerous tumor cells. (McDonald et al., 2008; Choi et al., 2010; Abbad et al., 2015; Li et al., 2014; Leung et al., 2010). Therefore, we studied the nanogel 

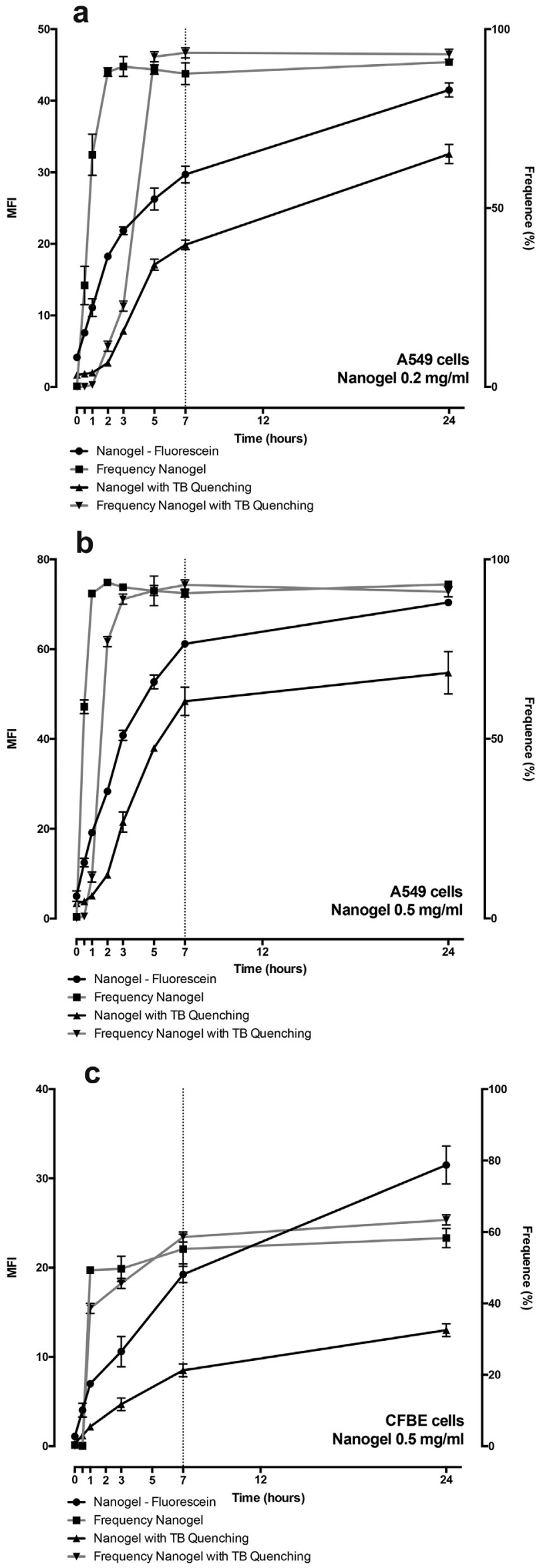

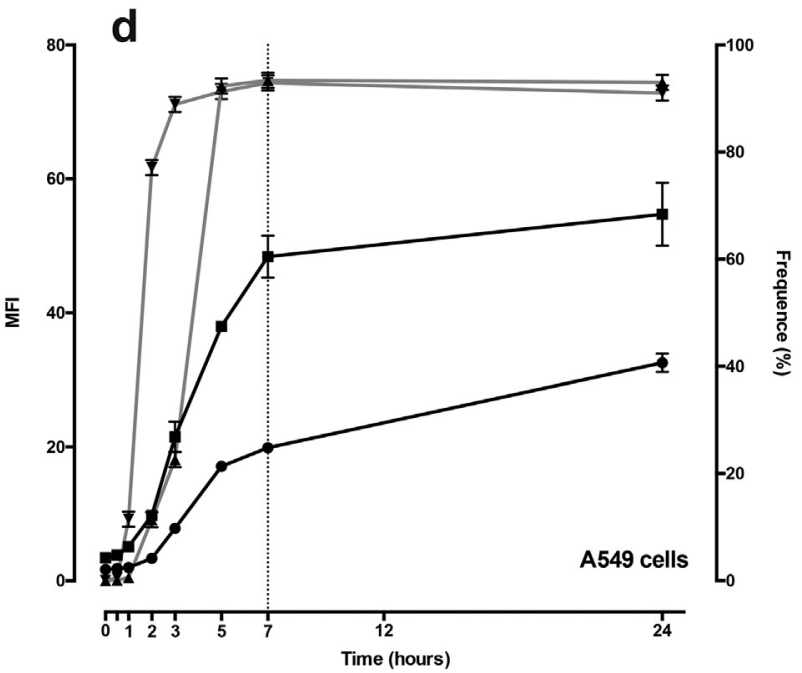

$\rightarrow 0.2 \mathrm{mg} / \mathrm{ml}$ Nanogel with TB Quenching

- $0.5 \mathrm{mg} / \mathrm{ml}$ Nanogel with TB Quenching

$\star 0.2 \mathrm{mg} / \mathrm{ml}$ Frequency

$\rightarrow 0.5 \mathrm{mg} / \mathrm{ml}$ Frequency

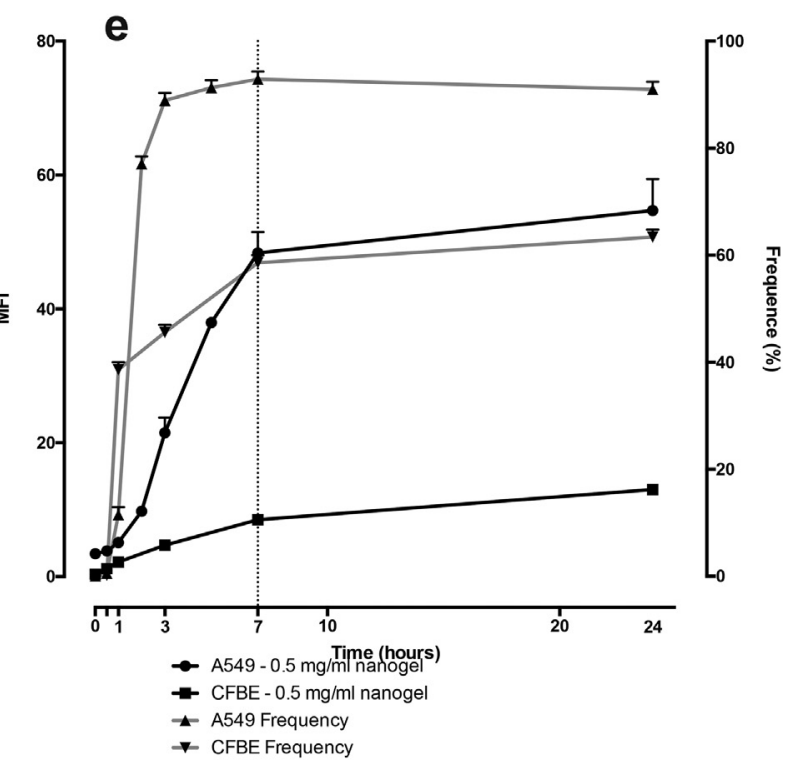




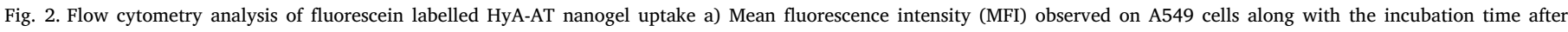

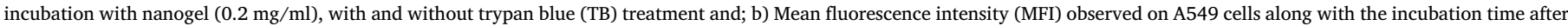

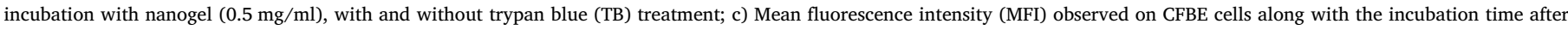

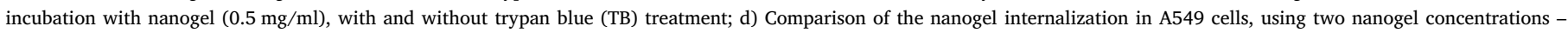

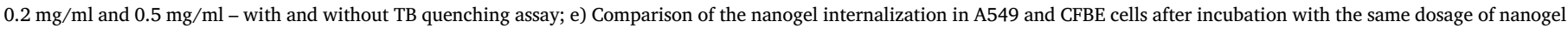

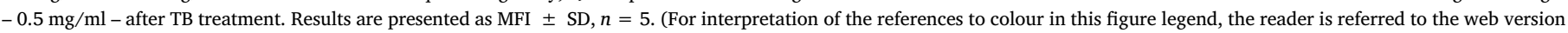
of this article.)

cellular uptake by A549 human non-small cancer lung cells that overexpress CD44 receptors. Also, cellular uptake was compared to CFBE cells, also obtained from pulmonary tissue (human cystic fibrosis), that do not overexpress those receptors (Reeves et al., 2011; Reeves et al., 2010; Vandivier et al., 2009).

Hence, A549 cells were treated with two nanogel concentrations $(0.2 \mathrm{mg} / \mathrm{ml}$ and $0.5 \mathrm{mg} / \mathrm{ml})$ to evaluate the dose effect on the internalization kinetics. In Fig. 2a, b, and d, we can see the nanogel uptake was dose dependent at almost all points, and fluorescence intensity was double.

We also analysed the nanogel uptake in CFBE cells (Fig. 2c). Although testing only the highest nanogel dosage we can see that MFI values were much lower than those obtained with A549 cells using similar conditions.

The TB exclusion assay makes it possible to distinguish between cell adhering and cell internalized nanogel particles. Trypan blue is a cell impermeable dye able to quench fluorescence signal originated from nanogel adhered to the cell surface (Vranic et al., 2013; Avelar-Freitas et al., 2014; Van Amersfoort and Van Strijp, 1994). Results of TB quenching, show that around $20-50 \%$ of the nanogel detected was not internalized (Fig. 2a, b and c).

An interesting feature of the internalization process in A549 cells concerns is its kinetics. As observed after quenching of membrane adherent nanogel in A549 cells, the rate of internalization was initially slow, and then accelerated considerably. This effect was more evident at the lowest dosage of nanogel.

Indeed, it has been reported that hyaluronic acid nanoparticles uptake by CD44 expressing cells may take 20-30 min (Schuldt, 2000; Scarselli and Donaldson, 2009) or up to $1 \mathrm{~h}$ (Zaki et al., 2011).

A comparative analysis of nanogel uptake in A549 and CFBE cells, after TB quenching revealed (Fig. 2e) a higher nanogel uptake in A549 cells, persuading us to think that nanogel uptake in these cells is mediated by HyA receptors and, probably by CD44 receptors - overexpressed in these cells.

Confocal microscopy analysis of nanogel internalization was also performed in A549 and CFBE cells, qualitatively corroborating the FCM results. In Fig. 3, we can see much brighter green fluorescence signals in the cytoplasm of A549 cells than, under the same conditions, in CFBE cells. To evaluate the mechanism of cellular uptake, a competitive study in which, A549 cells were treated with an excess of free HyA, $1 \mathrm{~h}$ before nanogel incubation was performed. As expected, fluorescence in HyAtreated A549 cells decreased substantially in comparison to non-treated cells. This result suggests that native HyA, may be interacting with cell HyA receptors - probably CD44 receptors - and inhibiting nangoel internalization by competitive cellular uptake.

\section{In vivo imaging studies}

\subsection{Nanogel labelling and characterization}

In order to evaluate the biodistribution profile of HyA-AT nanogel, Alexa680 and Cy5.5 were chemically conjugated to nanogel and native HyA (Fig. 4). Also, the biodistribution of each probe was analysed, to evaluate whether it bears specific affinity for a specific tissue. The obtained nanogel- probe conjugates were characterized regarding their size distribution profile, by DLS (Fig. 4). For biodistribution studies near-infrared (NIR) probes are a pertinent choice due to deep tissue penetration, high fluorescence signal and amenable self-quenching
(Ogawa et al., 2009). Cyanine dyes, and in particular 5.5, has shown high background fluorescence, high plasma protein binding and undesired aggregation. In turn, Alexa680 has higher excitation/emission threshold and therefore less background, being less sensitive to photobleaching (Luo et al., 2011; Yang et al., 2010).

\subsection{Comparison of Alexa Fluor 680 and Cy 5.5 pharmacokinetics in healthy mice}

Changes in size, surface charge, texture and heterogeneity in nanoparticles formulation are known to influence their biodistribution (Adjei et al., 2014). As shown in Fig. 4, the mean size diameter of the nanogel-probe conjugates was slightly higher in the nanogel-Cy5.5 conjugate. The zeta potential was also measured in the nanogels decorated with the probes and values of $-28.7 \mathrm{mV} \pm 1.99 \mathrm{mV}$ (Cy5.5), and $-18.9 \pm 1.87 \mathrm{mV}$ (Alexa680) were obtained. The original - non-labelled - nanogel zeta potential is $-19.3 \pm 1.97 \mathrm{mV}$ (Pedrosa et al., 2014). So it was not strange to see that, Cy5.5 conjugation increased somewhat the zeta potential of the nanogel, owing to its negative charge. Alexa680, on the other hand is a smaller, non-charged molecule (Fig. 4). The effect on the zeta potential suggests that the grafted Cy5.5 molecule, which reacts with the HyA carboxylic groups, is displayed on the nanogel surface. The Alexa680 grafting took place in the sulfhydryl residues of the hydrophobic chain of the nanogel and, also because of its lipophilicity, this probe may likely be lodged in nanogel hydrophobic domains. Since we could not find Alexa Fluor dyes reactive towards carboxylic groups, such as in the case of Cy5.5, we could not compare the two probes grafted using the same chemistry.

We studied the biodistribution of nanogel labelled with two different probes and as a control, native HyA and free probe. As can be seen in Fig. 5.Ia and .IIa, even after $48 \mathrm{~h}$ there was higher NIR background signal in Cy5.5 treated animals than in Alexa680 treated animals. In the later, signal was more concentrated and not spread in the whole body as seen for Cy5.5. The ex vivo images (Fig. 5.IIb) of major organs collected $48 \mathrm{~h}$ post-administration, revealed that Cy5.5 labelled samples showed a higher accumulation in lung, kidneys and skin, whereas, Alexa680 samples were mostly distributed to liver, kidneys and skin (Fig. 5.Ib). Adjei et al., 2014 compared the biodistribution profile of large anionic as opposed to small neutral, PLGA nanoparticles. They reported that large anionic NPs showed greater accumulation in the reticuloendothelial system, such as the liver and spleen, whereas small neutral NPs, showed accumulation in highly vascularized organs, such as the lungs, the kidneys and the heart. However, the mechanism throughout this occurs is yet to be proven. It is well established that the properties of the NPs interface influence protein binding and therefore their behaviour in vivo (Gref et al., 2000). One may speculate that the NIR probes can cause different protein corona in vivo upon entering the circulatory system and therefore affect, the organ distribution and accumulation. It has been reported that higher liver, spleen and bone marrow uptake is largely attributed to macrophages uptake. The serum proteins that compose the protein corona of nanomaterials are responsible for the recognition by the scavenger receptors of the macrophages (Kumar et al., 2010). Noteworthy is the fact that, for both probes, a low spleen accumulation was observed. Other researchers have also found a preferable lung accumulation when using Cy5.5 as NIR probe for pharmacokinetics studies. Hue et al., 2013 studied the ex vivo kinetic of free Cy5.5, and Cy5.5 labelled thermally cross-linked superparamagnetic iron oxide nanopar- 
Fluoresceín
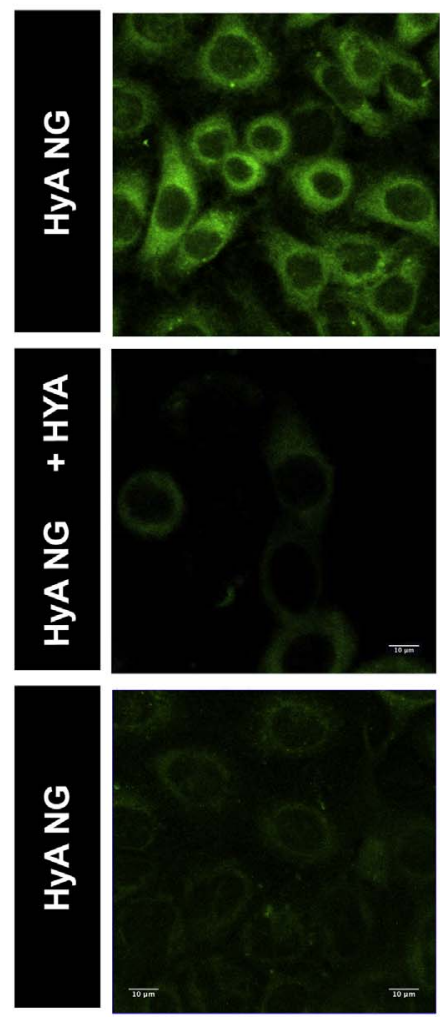

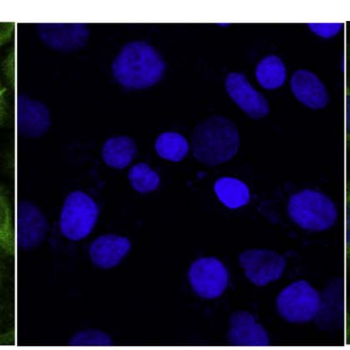

DAPI Merged
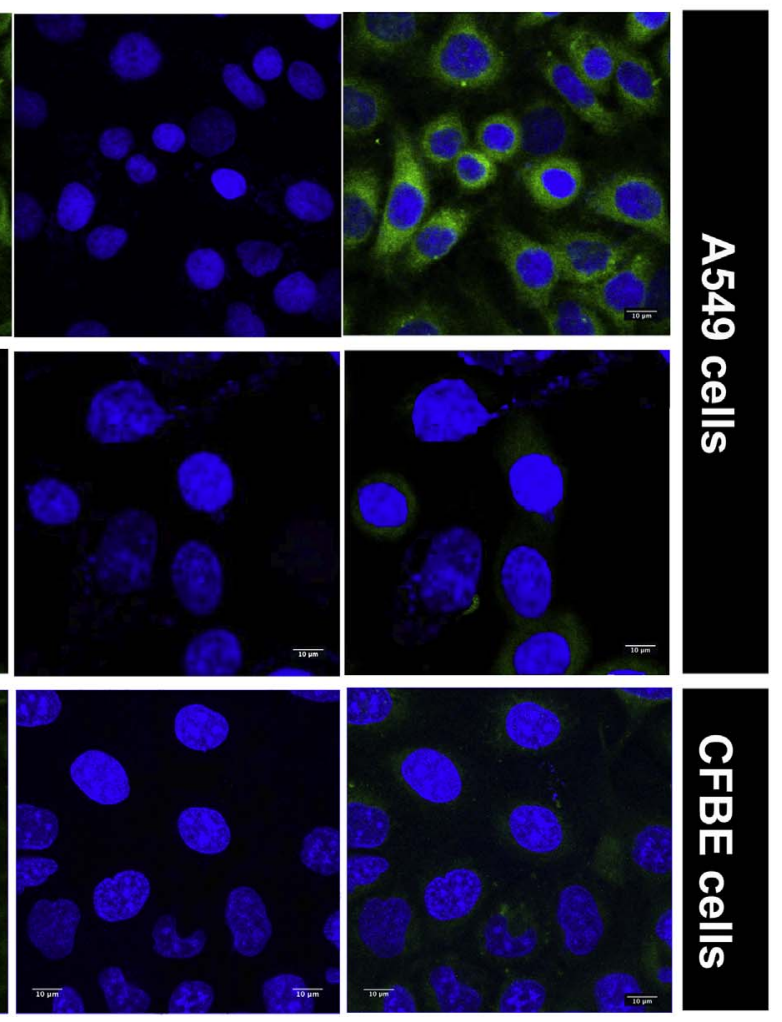

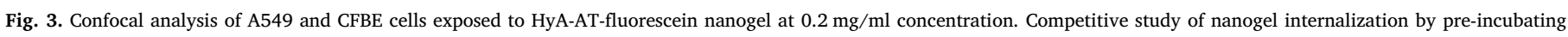

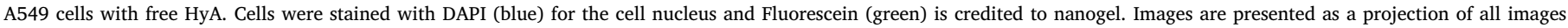
acquired in a Z stack. (For interpretation of the references to colour in this figure legend, the reader is referred to the web version of this article.)

ticles. Cy5.5 dye fluorescence in the body was rapidly eliminated, although high fluorescence intensity was observed in the lungs, kidneys and also liver. Thermally cross-linked superparamagnetic iron oxide nanoparticles labelled with Cy5.5, had the highest fluorescence intensity in the lungs at earlier times and decreased over time, until 28 days. Other researchers (Lee et al., 2012), have found that $7 \mathrm{~h}$ after administration Cy5.5 N-hydroxysuccinimide ester accumulated in the lungs and liver, and Cy5.5-conjugated $\mathrm{ZnO}$ nanoparticles showed strong signal in the kidneys and liver. Others researchers had encountered lung accumulation of nanoparticles when using Cy5.5 probe (Lee et al., 2007; Iqbal et al., 2010). Alexa680 biodistribution is at the moment not much studied and therefore, information regarding its pharmacokinetics is yet insufficient. Given the differential biodistribution of the nanogels labelled with the two NIR probes, it may be concluded that at least one of the probes influences the results obtained. Considering the published evidence suggesting that Cy5.5 exhibits some trend towards concentration in the lung, and the fact that in this case the probe is likely present in the surface of the particles (as opposed to the case of Alexa), we believe that the biodistribution obtained using Alexa680 is probably the more reliable one, therefore this was analysed with more detail.

Analysing Fig. 5.Ia, we could see that animals administered with nanogel exhibited higher fluorescence intensity at all time points, as compared to native HyA and Alexa680. In fact, $8 \mathrm{~h}$ after administration, free Alexa680 has almost fully cleared from animals and native HyA has taken an abrupt reduction. To further investigate sample biodistribution, we collected mice whole blood and major organs - spleen, heart, kidneys, lungs, liver, skin, muscle and brain - which were analysed ex vivo by NIRF spectroscopy (Fig. 5.Ib), since whole body images may be misleading (Choi et al., 2010; Choi et al., 2011; Yoon et al., 2013).

The results revealed that, $48 \mathrm{~h}$ after administration, only nanogel treated mice exhibited fluorescence in all organs, including the blood.
As described by others (Ogawa et al., 2009), Alexa Fluor is a small, neutral molecule that doesn't accumulate in any specific organ and is quickly eliminated from the body. A similar behaviour was observed in native HyA labelled samples. Indeed, after only $1 \mathrm{~h}$, HyA and free Alexa680 samples were almost extinguished. Researchers (Fraser et al., 1984; Necas et al., 2008) have described that native HyA half-life ranged between 2.5 and $5.5 \mathrm{~min}$. Also, described that its elimination predominantly by the kidneys, being the upper molecular weight limit for renal excretion, 25,000 Da. In turn, nanogel was concentrated in major organs, especially in the liver and kidneys, presumably in the reticuloendothelial system. Also, It has been demonstrated that (Kumar et al., 2010) smaller sized nanoparticles can be effectively cleared from body by renal excretion. Larger sized particles are processed by the reticuloendothelial system in the liver and kidneys. Nanogel has a bimodal size distribution, bearing two populations with $\sim 30 \mathrm{~nm}$ and $\sim 200 \mathrm{~nm}$, therefore it is not surprising that the nanogel is being filtered in the kidneys and internalized by macrophages in the liver (Choi et al., 2010; Arami et al., 2015). In addition, being made of hyaluronic acid, the nanogel is probably also digested by hyaluronidases to some extent.

\subsection{Biodistribution profile of nanogel in tumor xenograft models}

Understanding the in vivo biodistribution of nanogel, especially in disease animal models, is essential for the design and effective biomedical application of nanomedicines (Choi et al., 2010). Real-time NIRF images of nanogel and native HyA labelled with Alexa680, were assessed in A549 tumor-bearing mice, over the course of $48 \mathrm{~h}$. In vitro results demonstrated higher recognition and uptake of the HyA nanogel by A549 cells, which overexpress CD44 receptors. Therefore, we evaluated the HyA receptors targeting in mice subcutaneously induced with A549 tumor (Ganesh et al., 2013; Hwang et al., 2008).

As expected, initially a strong NIR signal was observed throughout 


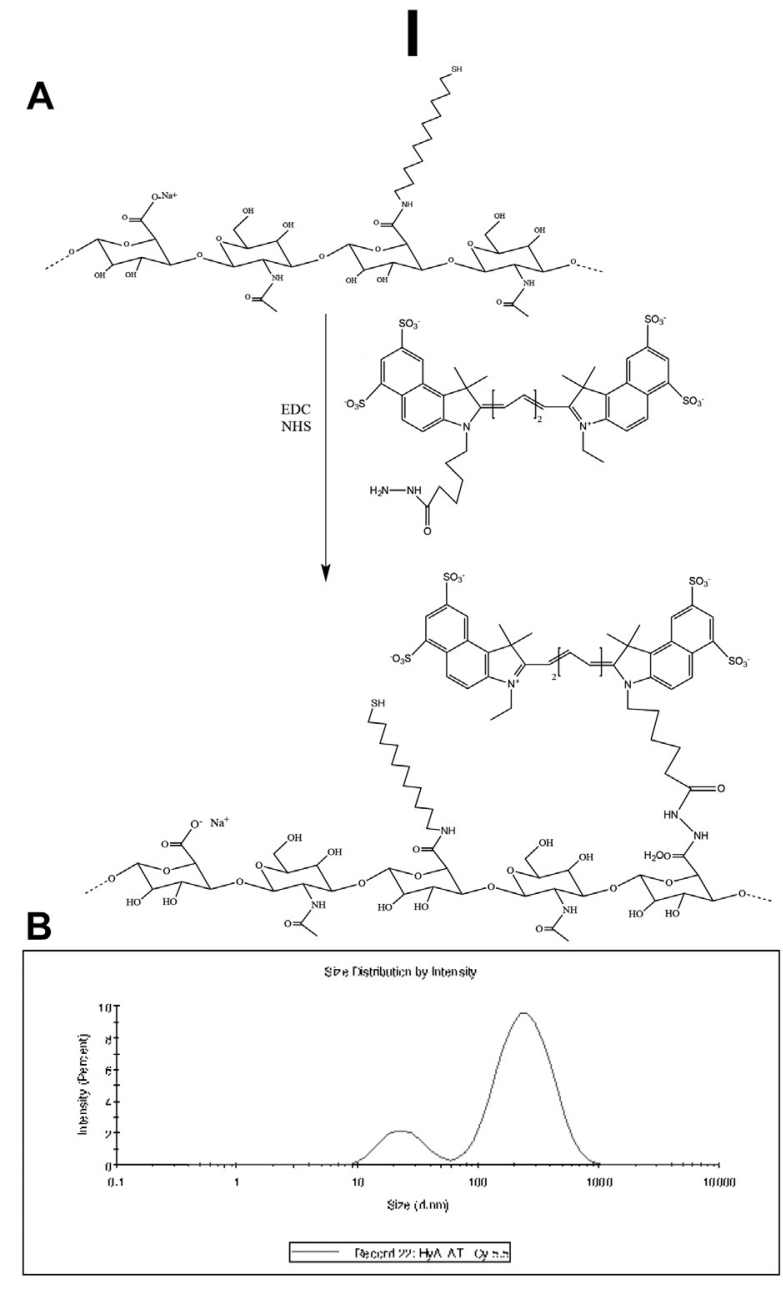

Size: $112.4 \mathrm{~nm}$

Pdl: 0.344

C

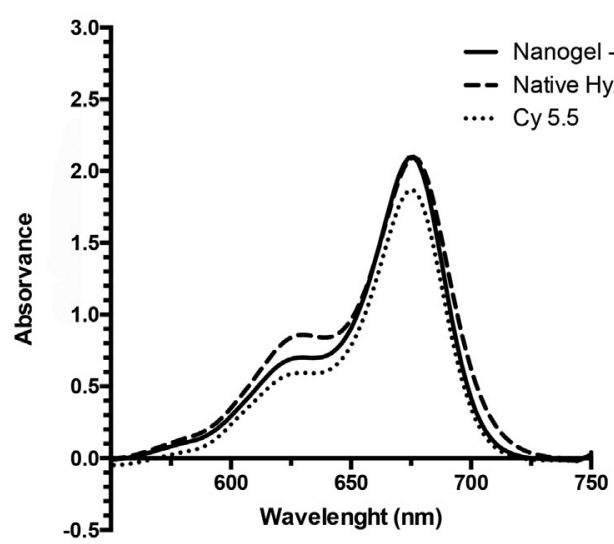

II

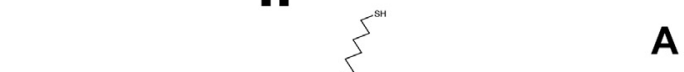

A

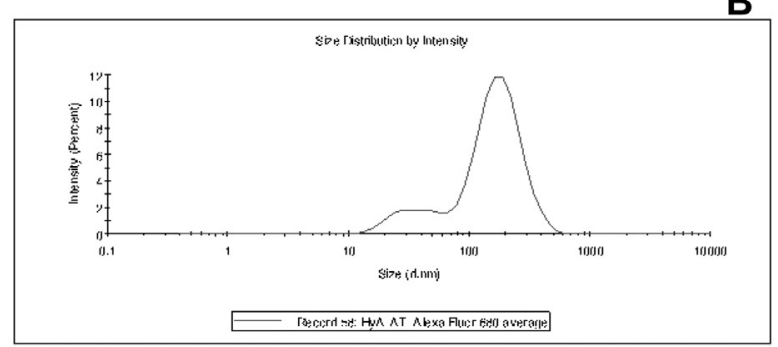

Size: $98.35 \mathrm{~nm}$

Pdl: 0.396

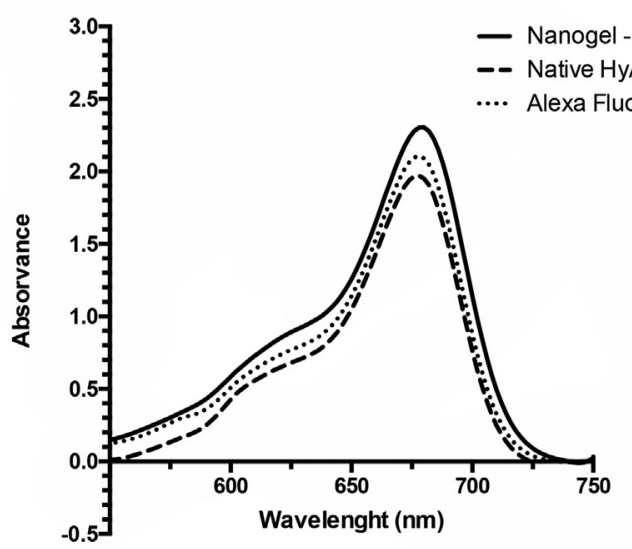

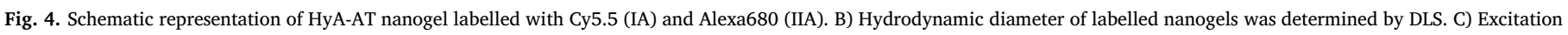
spectra of $4 \times$ diluted HyA-AT-Cy5.5 and HyA-AT-Alexa680 samples.

the body (Fig. 6.I), in the major organs and blood (Fig. 6.II) after administration of both nanogel and native HyA samples. As expected, and as observed in the case of the healthy animals, liver was the major clearance organ. Only $5 \mathrm{~min}$ after administration, NIR images revealed similar distribution for nanogel and HyA, namely in tumor tissue. In turn, $1 \mathrm{~h}$ post-administration, the free polymer signal decreased noticeably in all organs and largely in the tumor, while the nanogel concentrated over the first hour at tumor site (Fig. 6.V). Around $8 \mathrm{~h}$ post-administration it was noticeable that almost all traces of HyA were eliminated. However, at that time point, the nanogel remained detectable in the tumor, exhibiting a strong signal. This effect may be due to a combination of two factors; receptor mediated targeting through CD44 and enhanced permeation and retention (EPR) (Ganesh et al., 2013; Leung et al., 2010; Whitmire et al., 2012; Bartlett et al., 2007; Yhee 
a
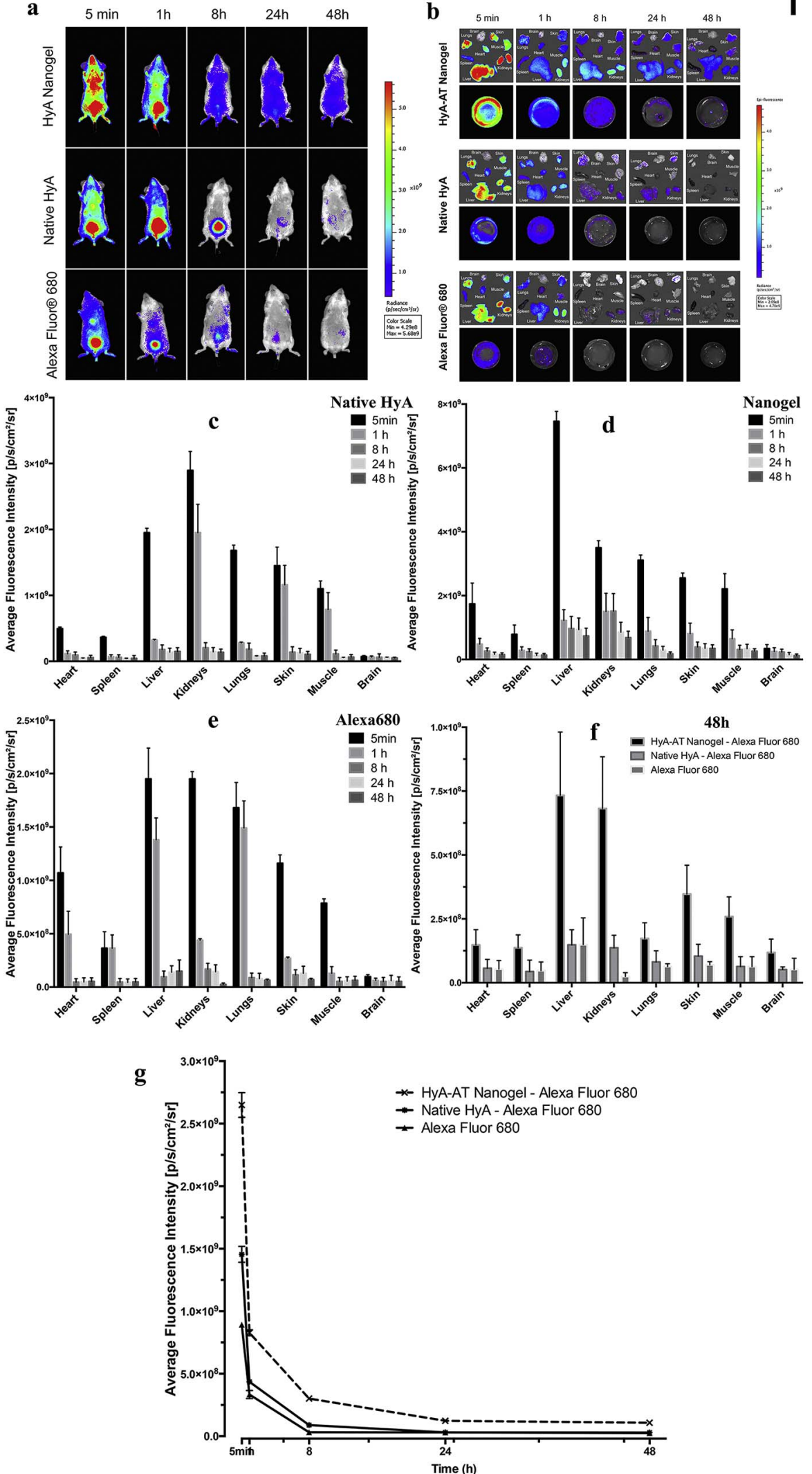

$48 \mathrm{~h}$
I
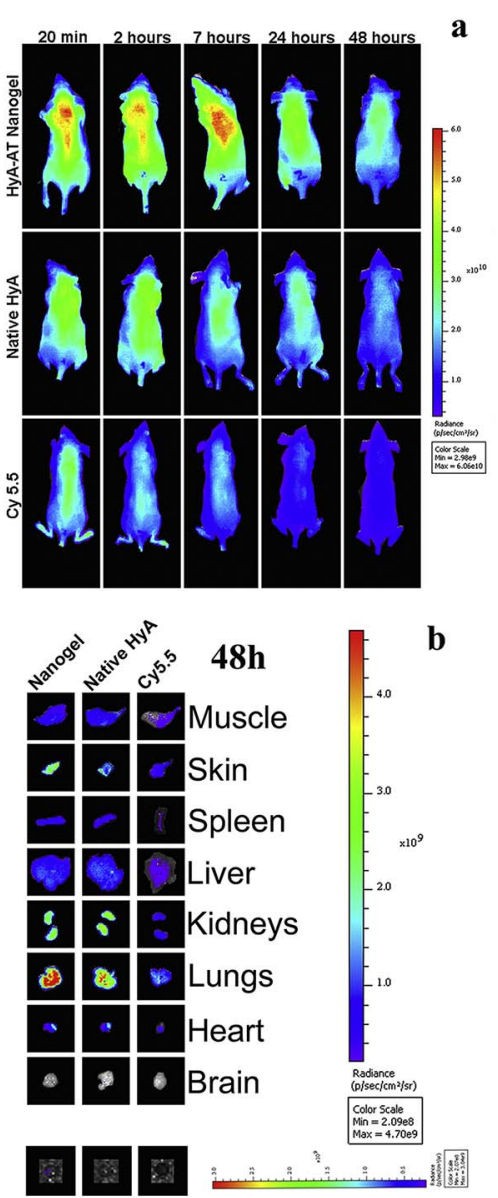

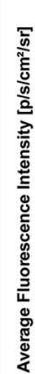
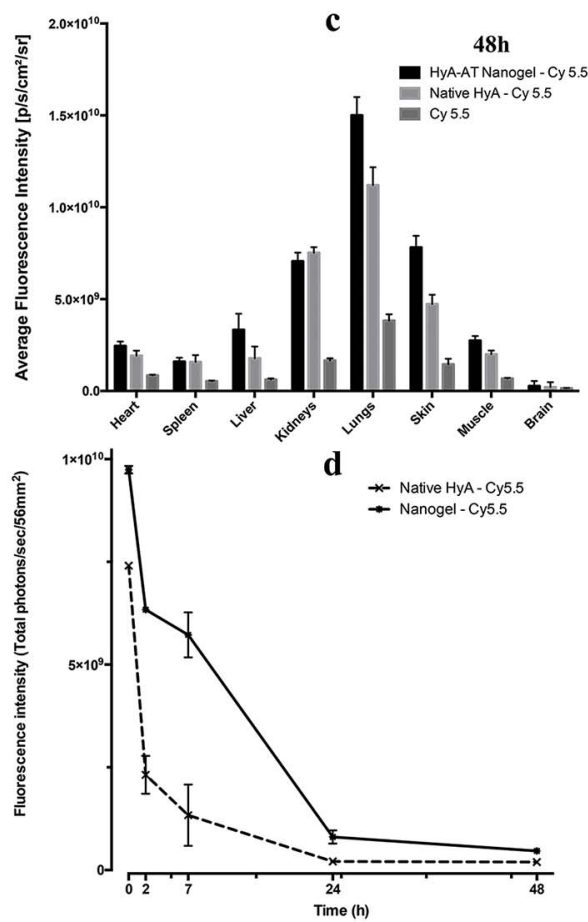

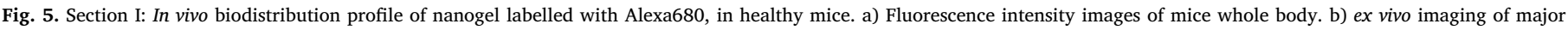

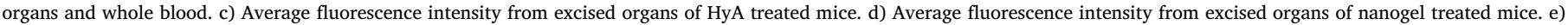

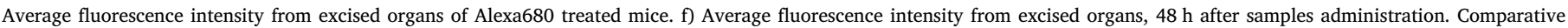

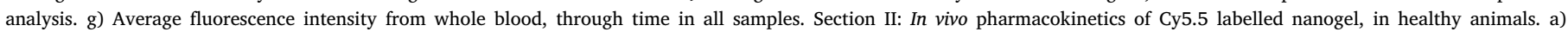

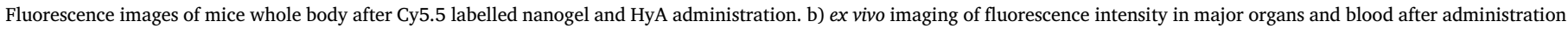

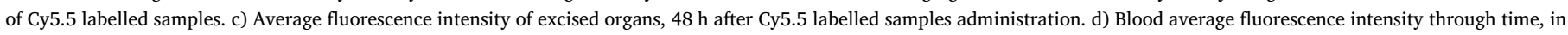
Cy5.5 labelled samples. Data are shown as mean $\pm \mathrm{SD}, n=5$. 
I

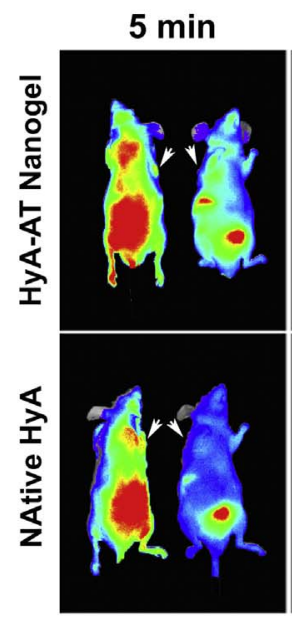

$1 \mathrm{~h}$

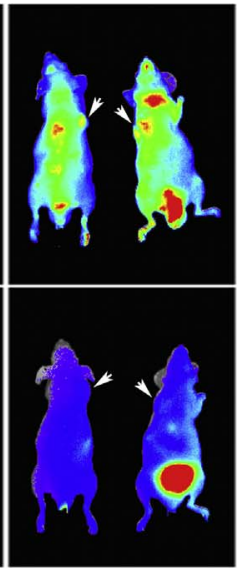

$8 \mathrm{~h}$

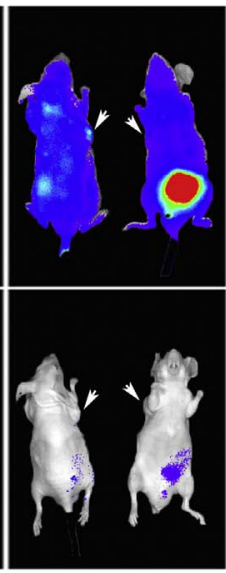

$24 \mathrm{~h}$

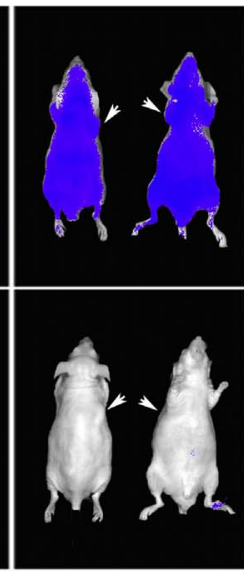

$48 \mathrm{~h}$

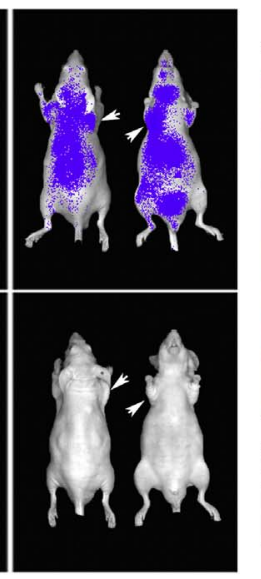

Epi-fiuorescence

E 1.4

$E^{1.2}$

$E^{0.8} \times 10^{10}$

$E^{E^{0.6}}$

Radiance
(p/sec/ $/ \mathrm{cm}^{2} / \mathrm{sr}$ )

\begin{tabular}{|l|}
\hline Color Scale \\
Min $=1.84 \mathrm{eg}$
\end{tabular}

\begin{tabular}{l} 
Min $=1.84 \mathrm{eg}$ \\
$\operatorname{Max}=1.46 \mathrm{e} 10$ \\
\hline
\end{tabular}

II
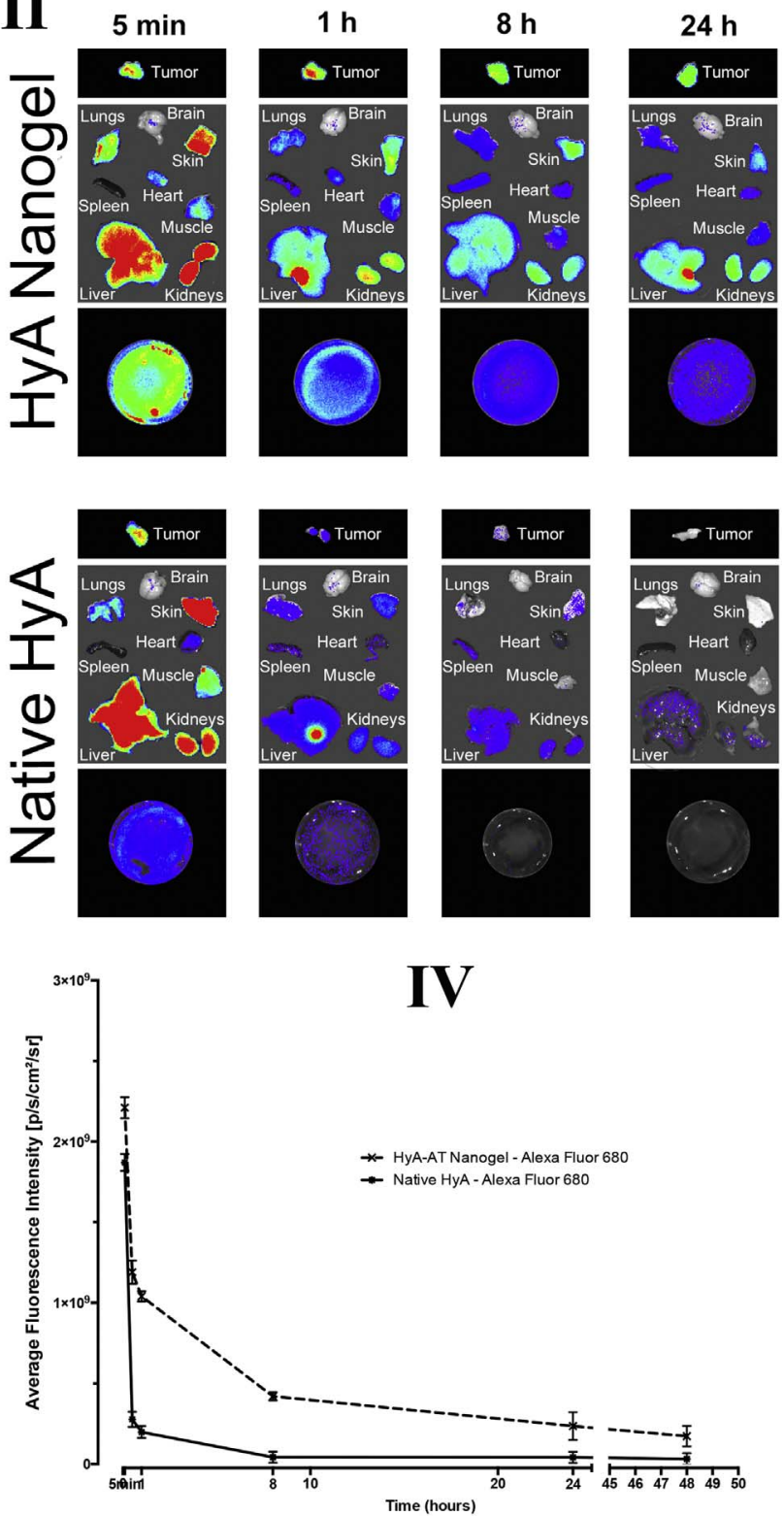
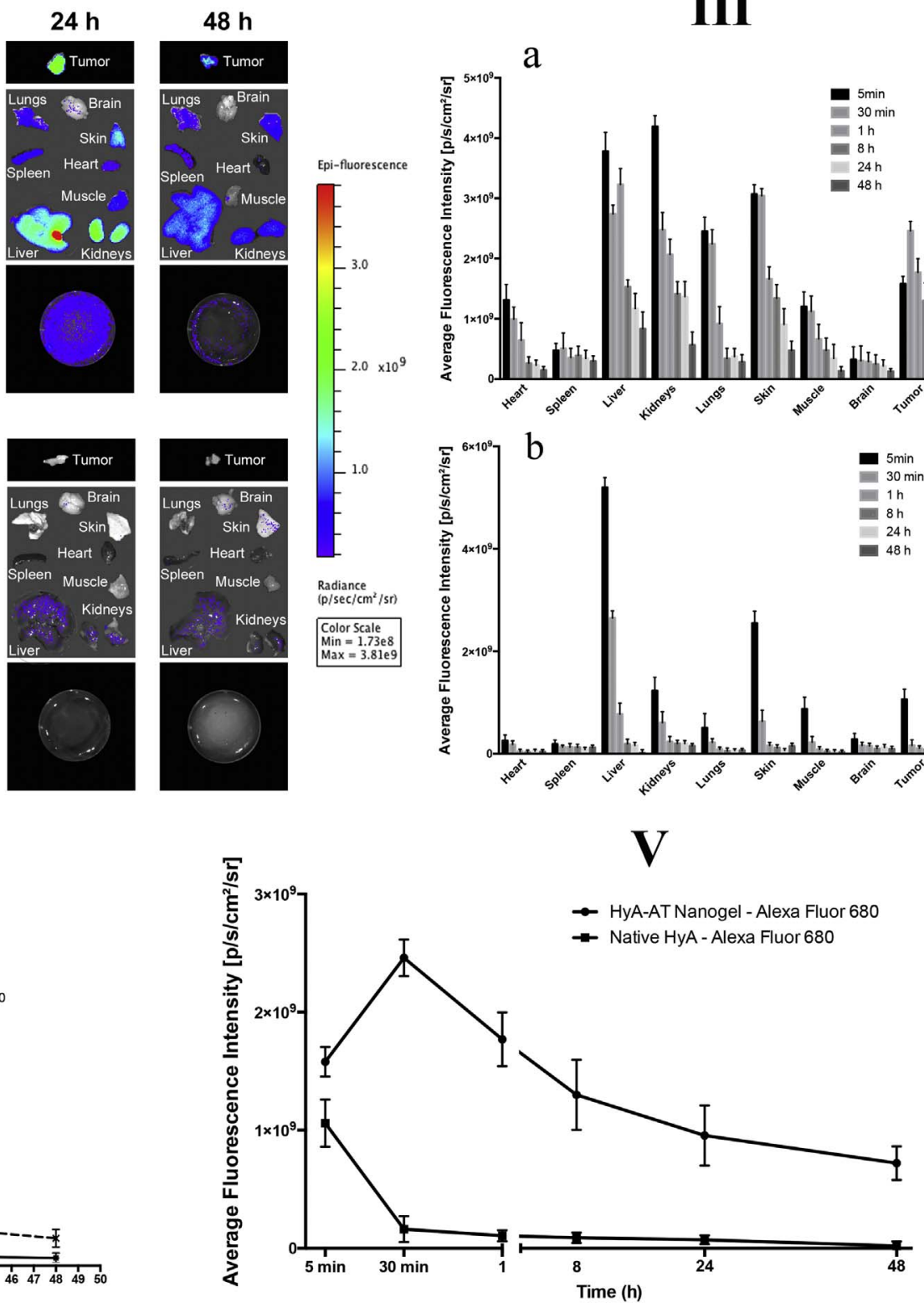

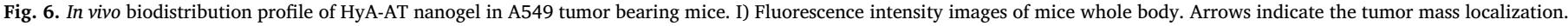

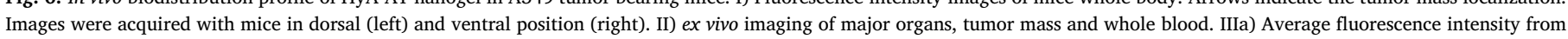

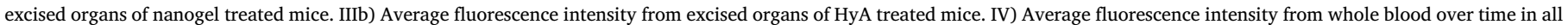
samples. V) Average fluorescence intensity in tumor mass over time in all samples. Data are shown as mean $\pm \mathrm{SD}, n=5$. 
et al., 2014). Also, the fact that nanogel continued to be detected in circulation up to $48 \mathrm{~h}$ (Fig. 6.II and .IV) it is expected to allow higher tumor targetability. The increased blood circulation half-life, as well as the longer residence time in the tumor tissue as compared to the native hyaluronic acid, is probably due to the lower degradability owing to the chemical modification. Ganesh et al., also described in vivo high tumor targetability in A549 cell lines (Ganesh et al., 2013), using cisplatin loaded CD44 targeted hyaluronic acid nanoparticles. Photo-crosslinked hyaluronic acid nanoparticles (Yoon et al., 2013) and hyaluronic acid nanoparticles (Choi et al., 2010) also demonstrated hyaluronic acid targetability to SCC7 cancer cells, also CD44 overexpressing cells.

\section{Conclusion}

Hyaluronic acid nanogel showed in vitro and in vivo targetability towards HyA receptors, probably associated with CD44 receptors. In vitro studies revealed higher cellular uptake by CD44 overexpressing lung cancer cells. Also, in vivo reports showed selective targeting towards tumor tissue, probably due to both passive accumulation through EPR effect and active targeting by HyA receptor affinity. It is described that nanomaterials behaviour in vivo, namely its pharmacokinetics, is influenced by size, shape and surface characteristics; in this work we wanted to observe the influence of the NIR probe used for biodistribution studies. The most studied probe in NIR imaging is Cy5.5. Therefore, we wanted to compare the pharmacokinetics profile of nanogel labelled with Cy5.5 and Alexa680 with similar excitation and emission wavelength. Our results showed that organ accumulation was different in nanogel labelled with Cy5.5 and Alexa680. Further work, regarding the protein corona of labelled nanogel is necessary to understand the differences in pharmacokinetics profiles. In conclusion, results show that HyA-AT nanogel can be used for active tumor targeting, as a drug delivery system, optical imaging agent and others.

\section{Acknowledgments}

The authors thank the FCT Strategic Project of UID/BIO/04469/ 2013 unit, the project RECI/BBB-EBI/0179/2012 (FCOMP-01-0124FEDER-027462) and the Project "BioHealth - Biotechnology and Bioengineering approaches to improve health quality", Ref. NORTE07-0124-FEDER-000027, co-funded by the Programa Operacional Regional do Norte (ON.2 - O Novo Norte), QREN, FEDER. We would like to acknowledge also the support of FCT for the PhD grant reference SFRH/BD/61516/2009. We would also like to thank Bioimaging department on Molecular Medicine Institute (IMM) in Lisbon, namely Dr. José Rino and Dr. António Temudo. Also thank the animal facilities in IMM (Lisbon), specially Dra. Dolores Bonaparte and Dra. Joana Marques. Also, we would like to acknowledge the special contribution of Professor Fatima Baltazar in ICVS (Braga) for kindly grant us CFBE and A549 cell lines.

\section{References}

Abbad, S., et al., 2015. Preparation, in vitro and in vivo evaluation of polymeric nanoparticles based on hyaluronic acid-poly(butyl cyanoacrylate) and D-alphatocopheryl polyethylene glycol 1000 succinate for tumor-targeted delivery of morin hydrate. Int. J. Nanomedicine 10, 305-320.

Adjei, I.M., Peetla, C., Labhasetwar, V., 2014. Heterogeneity in nanoparticles influences biodistribution and targeting. Nanomedicine (London) 9 (2), 267-278.

Ahn, B., Rhee, S.G., Stadtman, E.R., 1987. Use of fluorescein hydrazide and fluorescein thiosemicarbazide reagents for the fluorometric determination of protein carbonyl groups and for the detection of oxidized protein on polyacrylamide gels. Anal. Biochem. 161 (2), 245-257.

Arami, H., et al., 2015. In vivo delivery, pharmacokinetics, biodistribution and toxicity of iron oxide nanoparticles. Chem. Soc. Rev.

Arpicco, S., et al., 2014. Hyaluronic acid conjugates as vectors for the active targeting of drugs, genes and nanocomposites in cancer treatment. Molecules 19 (3), 3193-3230.

Avelar-Freitas, B.A., et al., 2014. Trypan blue exclusion assay by flow cytometry. Braz. J. Med. Biol. Res. 47 (4), 307-315.

Bae, K.H., Mok, H., Park, T.G., 2008. Synthesis, characterization, and intracellular delivery of reducible heparin nanogels for apoptotic cell death. Biomaterials 29 (23),
3376-3383.

Bae, K.H., et al., 2009. Intracellular delivery of heparin complexed with chitosan-g-poly (ethylene glycol) for inducing apoptosis. Pharm. Res. 26 (1), 93-100.

Bartlett, D.W., et al., 2007. Impact of tumor-specific targeting on the biodistribution and efficacy of siRNA nanoparticles measured by multimodality in vivo imaging. Proc. Natl. Acad. Sci. U. S. A. 104 (39), 15549-15554.

Berlier, J.E., et al., 2003. Quantitative comparison of long-wavelength Alexa Fluor dyes to Cy dyes: fluorescence of the dyes and their bioconjugates. J. Histochem. Cytochem. 51 (12), 1699-1712.

Choi, K.Y., et al., 2010. Self-assembled hyaluronic acid nanoparticles for active tumor targeting. Biomaterials 31 (1), 106-114.

Choi, K.Y., et al., 2011. Smart nanocarrier based on PEGylated hyaluronic acid for cancer therapy. ACS Nano 5 (11), 8591-8599.

Fotakis, G., Timbrell, J.A., 2006. In vitro cytotoxicity assays: comparison of LDH, neutral red, MTT and protein assay in hepatoma cell lines following exposure to cadmium chloride. Toxicol. Lett. 160 (2), 171-177.

Fraser, J.R., et al., 1984. Elimination of hyaluronic acid from the blood stream in the human. Clin. Exp. Pharmacol. Physiol. 11 (1), 17-25.

Ganesh, S., et al., 2013. In vivo biodistribution of siRNA and cisplatin administered using CD44-targeted hyaluronic acid nanoparticles. J. Control. Release 172 (3), 699-706.

Goodarzi, N., et al., 2013. A review of polysaccharide cytotoxic drug conjugates for cancer therapy. Carbohydr. Polym. 92 (2), 1280-1293.

Gratton, S.E., et al., 2008. The effect of particle design on cellular internalization pathways. Proc. Natl. Acad. Sci. U. S. A. 105 (33), 11613-11618.

Gref, R., et al., 2000. 'Stealth' corona-core nanoparticles surface modified by polyethylene glycol (PEG): influences of the corona (PEG chain length and surface density) and of the core composition on phagocytic uptake and plasma protein adsorption. Colloids Surf. B: Biointerfaces 18 (3-4), 301-313.

Gruber, H.J., et al., 2000. Anomalous fluorescence enhancement of Cy3 and Cy3.5 versus anomalous fluorescence loss of Cy5 and Cy7 upon covalent linking to IgG and noncovalent binding to avidin. Bioconjug. Chem. 11 (5), 696-704.

Hermanson, G.T. (Ed.), 2008. Bioconjugate Techniques, second ed. Academic Press, San Diego, pp. xxx (1202 pp.).

Hue, J.J., et al., 2013. Distribution and accumulation of Cy5.5-labeled thermally crosslinked superparamagnetic iron oxide nanoparticles in the tissues of ICR mice. J. Vet. Sci. 14 (4), 473-479.

Hwang, H.Y., et al., 2008. Tumor targetability and antitumor effect of docetaxel-loaded hydrophobically modified glycol chitosan nanoparticles. J. Control. Release 128 (1), 23-31.

Ilgin, P., et al., 2010. Colloidal drug carries from (sub)micron hyaluronic acid hydrogel particles with tunable properties for biomedical applications. Carbohydr. Polym. 82 (3), 997-1003.

Iqbal, U., et al., 2010. Molecular imaging of glioblastoma multiforme using anti-insulinlike growth factor-binding protein-7 single-domain antibodies. Br. J. Cancer 103 (10), 1606-1616.

Kadi, S., et al., 2009. Alkylamino hydrazide derivatives of hyaluronic acid: synthesis, characterization in semidilute aqueous solutions, and assembly into thin multilayer films. Biomacromolecules 10 (10), 2875-2884.

Keogh, M.J., et al., 2011. Simultaneous measurement of phagocytosis and respiratory burst of leukocytes in whole blood from bottlenose dolphins (Tursiops truncatus) utilizing flow cytometry. Vet. Immunol. Immunopathol. 144 (3-4), 468-475.

Kumar, R., et al., 2010. In vivo biodistribution and clearance studies using multimodal organically modified silica nanoparticles. ACS Nano 4 (2), 699-708.

Lee, H., et al., 2007. Thermally cross-linked superparamagnetic iron oxide nanoparticles: synthesis and application as a dual imaging probe for cancer in vivo. J. Am. Chem. Soc. 129 (42), 12739-12745.

Lee, C.M., et al., 2012. Optical imaging to trace near infrared fluorescent zinc oxide nanoparticles following oral exposure. Int. J. Nanomedicine 7, 3203-3209.

Leung, E.L., et al., 2010. Non-small cell lung cancer cells expressing CD44 are enriched for stem cell-like properties. PLoS One 5 (11), e14062.

Li, J., et al., 2014. Hyaluronic acid-modified hydrothermally synthesized iron oxide nanoparticles for targeted tumor MR imaging. Biomaterials 35 (11), 3666-3677.

Luo, Y., Prestwich, G.D., 1999. Synthesis and selective cytotoxicity of a hyaluronic acidantitumor bioconjugate. Bioconjug. Chem. 10 (5), 755-763.

Luo, S., et al., 2011. A review of NIR dyes in cancer targeting and imaging. Biomaterials 32 (29), 7127-7138.

McDonald, B., et al., 2008. Interaction of CD44 and hyaluronan is the dominant mechanism for neutrophil sequestration in inflamed liver sinusoids. J. Exp. Med. 205 (4), 915-927.

Mosmann, T., 1983. Rapid colorimetric assay for cellular growth and survival: application to proliferation and cytotoxicity assays. J. Immunol. Methods 65 (1-2), 55-63.

$\mathrm{Na}$, K., et al., 2007. Synergistic effect of TGFbeta-3 on chondrogenic differentiation of rabbit chondrocytes in thermo-reversible hydrogel constructs blended with hyaluronic acid by in vivo test. J. Biotechnol. 128 (2), 412-422.

Necas, J., et al., 2008. Hyaluronic acid (hyaluronan): a review. Vet. Med. 53 (8), $397-411$.

Nobs, L., et al., 2003. Surface modification of poly(lactic acid) nanoparticles by covalent attachment of thiol groups by means of three methods. Int. J. Pharm. 250 (2), $327-337$.

Ogawa, M., et al., 2009. In vivo target-specific activatable near-infrared optical labeling of humanized monoclonal antibodies. Mol. Cancer Ther. 8 (1), 232-239.

Pedrosa, S.S., et al., 2014. A novel crosslinked hyaluronic acid nanogel for drug delivery. Macromol. Biosci. 14 (11), 1556-1568.

Platt, V.M., Szoka Jr., F.C., 2008. Anticancer therapeutics: targeting macromolecules and nanocarriers to hyaluronan or CD44, a hyaluronan receptor. Mol. Pharm. 5 (4), 474-486. 
Reeves, E.P., et al., 2010. IL-8 dictates glycosaminoglycan binding and stability of IL-18 in cystic fibrosis. J. Immunol. 184 (3), 1642-1652.

Reeves, E.P., et al., 2011. The involvement of glycosaminoglycans in airway disease associated with cystic fibrosis. ScientificWorldJournal 11, 959-971.

Roudi, R., et al., 2014. CD44 and CD24 cannot act as cancer stem cell markers in human lung adenocarcinoma cell line A549. Cell Mol Biol Lett 19 (1), 23-36.

Scarselli, M., Donaldson, J.G., 2009. Constitutive internalization of G protein-coupled receptors and $\mathrm{G}$ proteins via clathrin-independent endocytosis. J. Biol. Chem. 284 (6), 3577-3585.

Schuldt, A., 2000. Nature reviews. In: Molecular Cell Biology. Nature Pub. Group, London, UK (p. v).

Tanzer, M.L., Monroe, D., Gross, J., 1966. Inhibition of collagen intermolecular crosslinking by thiosemicarbazide. Biochemistry 5 (6), 1919-1926.

Van Amersfoort, E.S., Van Strijp, J.A., 1994. Evaluation of a flow cytometric fluorescence quenching assay of phagocytosis of sensitized sheep erythrocytes by polymorphonuclear leukocytes. Cytometry 17 (4), 294-301.

Vandivier, R.W., et al., 2009. Dysfunctional cystic fibrosis transmembrane conductance regulator inhibits phagocytosis of apoptotic cells with proinflammatory consequences. Am. J. Phys. Lung Cell. Mol. Phys. 297 (4), L677-L686.

Vranic, S., et al., 2013. Deciphering the mechanisms of cellular uptake of engineered nanoparticles by accurate evaluation of internalization using imaging flow cytometry. Part. Fibre Toxicol 10, 2.

Whitmire, R.E., et al., 2012. Self-assembling nanoparticles for intra-articular delivery of anti-inflammatory proteins. Biomaterials 33 (30), 7665-7675.

Yang, X., et al., 2010. Near IR heptamethine cyanine dye-mediated cancer imaging. Clin. Cancer Res. 16 (10), 2833-2844.

Yhee, J.Y., Lee, S., Kim, K., 2014. Advances in targeting strategies for nanoparticles in cancer imaging and therapy. Nano 6 (22), 13383-13390.

Yoon, H.Y., et al., 2013. Photo-crosslinked hyaluronic acid nanoparticles with improved stability for in vivo tumor-targeted drug delivery. Biomaterials 34 (21), 5273-5280.

Zaki, N.M., Nasti, A., Tirelli, N., 2011. Nanocarriers for cytoplasmic delivery: cellular uptake and intracellular fate of chitosan and hyaluronic acid-coated chitosan nanoparticles in a phagocytic cell model. Macromol. Biosci. 11 (12), 1747-1760.

Zhang, H.Z., et al., 2010. The study of the tumor stem cell properties of CD133 + CD44 + cells in the human lung adenocarcinoma cell line A549. Cell. Mol. Biol. (Noisy-leGrand) 56, OL1350-OL1358 (Suppl.). 